\title{
Graphical Modelling for Brain Connectivity via Partial Coherence
}

\author{
T. Medkour ${ }^{\mathrm{a}}$, A. T. Walden ${ }^{\mathrm{a}, *}$, A. Burgess ${ }^{\mathrm{b}}$ \\ ${ }^{a}$ Department of Mathematics, Imperial College London, 180 Queen's Gate, London SWr 2BZ, UK \\ ${ }^{b}$ Aston University School of Life and Health Sciences, Aston Triangle, Birmingham B4 7ET, UK
}

\begin{abstract}
Spectral and coherence methodologies are ubiquitous for the analysis of multiple time series. Partial coherence analysis may be used to try to determine graphical models for brain functional connectivity. The outcome of such an analysis may be considerably influenced by factors such as the degree of spectral smoothing, line and interference removal, matrix inversion stabilization and the suppression of effects caused by side-lobe leakage, the combination of results from different epochs and people, and multiple hypothesis testing. This paper examines each of these steps in turn and provides a possible path which produces relatively 'clean' connectivity plots. In particular we show how spectral matrix diagonal upweighting can simultaneously stabilize spectral matrix inversion and reduce effects caused by side-lobe leakage, and use the stepdown multiple hypothesis test procedure to help formulate an interaction strength.
\end{abstract}

Key words: Brain connectivity, Graphical models, Partial spectral coherence

\section{Introduction}

A tenet of modern neuroscience, that the computational properties of the brain are a direct consequence of its circuitry, has created huge current interest in brain connectivity (Valdés-Sosa et al., 2005; Hämäläinen et al., 2005; Celka, 2005). With such an explosion of interest in brain connectivity research, it is timely to examine some of the important statistical issues affecting the use of partial coherence analysis in the determination of graphical models for brain functional connectivity. We shall focus on the analysis of EEG measurements representing cortical activity as potential, measured over the scalp of schizophrenic patients and controls. Our ultimate objective is to use graphical models to compare brain connectivity in these two groups. If as here, sources of 'driving' are not being investigated, and linear relationships between series are assumed, then partial coherence is a well-established frequency domain approach for constructing graphical models (Dahlhaus, 2000).

The vertices in a constructed graph represent the different scalp locations of the electrodes, while an edge between two vertices reflect a direct connection between the measured series at the two locations. The absence of an edge in the graphical model, i.e., the absence of a connection between series recorded at two sites on the scalp, is indicated by a corresponding null partial coherence for the two series. The ability to determine the absence of an edge is the key aspect of the construction of a meaningful graphical model.

${ }^{*}$ Corresponding author. Tel: (0)20 7594 8524; Fax: (0)20 7594 8517; e-mail: a.walden@imperial.ac.uk
Although of course statistical estimation would preclude estimated partial coherences from being exactly null, so that hypothesis testing is required, the practical situation is actually significantly more complicated. A serious problem with partial coherence is its stable estimation.

Suppose we have a set of $p>2$ time series. Partial coherence is simply the frequency domain expression of the squared partial or conditional linear correlation between two time series when the remaining $p-2$ series are held constant. Uses of partial coherence analysis in neuroscience tend to divide up into cases where the number, $p-2$, of conditioning variables (partial coherence "order") is small, and cases where it is large.

For example partial coherence has been used to investigate differential connectivity between neurons (Cohen et al., 1995), nerve-to-nerve coherence conditioned on arterial pulse (Larsen et al., 2000) and interhemispheric coherence with conditioning of the EEG signals by the occipital alpha rhythm (Mima et al., 2000); in each case the partial coherence order was unity. Rosenberg et al. (1989) looked at small networks of neurones to detect which neurones interact directly or are influenced by common inputs; they gave examples of partial coherences of order 2, extended in Rosenberg et al. (1998) to order 3, and to order 7 in Halliday (2005).

Salvador et al. (2005) produce, via partial coherence analysis, graphs of functional connectivity based on functional magnetic resonance imaging time series measured in $p=90$ cortical and subcortical regions of the brains of healthy volunteers.

When the partial coherence order is low, iterative schemes which calculate partial coherence directly from 
cross-spectra are viable. In this paper we are interested in a large partial coherence order and in such cases partial coherence is calculated from the inverse of the $p \times p$ spectral matrix, and well-defined issues arise which must be addressed regarding the stability of the inversion.

\section{Statistical background}

\subsection{Spectral matrix}

Here we consider a real-valued discrete time stochastic vector process $\left\{\boldsymbol{X}_{t}\right\}$ with $p$ component processes, whose $t$ th element is the column vector $\boldsymbol{X}_{t}=\left[X_{1, t}, \ldots, X_{p, t}\right]^{T}$, and each component process has zero mean. The sample interval is denoted $\Delta t$ and $f_{\mathcal{N}}=1 /(2 \Delta t)$ is the Nyquist frequency.

We assume the $p$ processes are jointly stationary. The spectral matrix is given by

$$
\boldsymbol{S}(f)=\left[\begin{array}{cccc}
S_{11}(f) & S_{12}(f) & \ldots & S_{1 p}(f) \\
S_{21}(f) & S_{22}(f) & \ldots & S_{2 p}(f) \\
\vdots & \vdots & \ddots & \vdots \\
S_{p 1}(f) & S_{p 2}(f) & \ldots & S_{p p}(f)
\end{array}\right]
$$

where the $l, m$ th term $S_{l m}(f)=(\boldsymbol{S}(f))_{l m}$ is the crossspectrum for the $l$ and $m$ th processes.

\subsection{Partial coherence}

Let $\left\{X_{j, t}\right\}$ and $\left\{X_{k, t}\right\}$ be two distinct component series in $\left\{\boldsymbol{X}_{t}\right\}$. The partial cross-spectral density function $S_{j k \mid(\backslash j k)}(f)$ of $\left\{X_{j, t}\right\}$ and $\left\{X_{k, t}\right\}$ is defined as

$$
S_{j k \mid(\backslash j k)}(f)=S_{j k}(f)-\boldsymbol{S}_{j(\backslash j k)}(f) \boldsymbol{S}_{(\backslash j k)(\backslash j k)}^{-1}(f) \boldsymbol{S}_{(\backslash j k) k}(f),
$$

where $(\backslash j k)$ means "all series except the $j$ th and $k$ th," i.e.,

$$
\begin{aligned}
\boldsymbol{S}_{j(\backslash j k)}(f)= & {\left[S_{j 1}(f), \ldots, S_{j(j-1)}(f), S_{j(j+1)}(f), \ldots,\right.} \\
& \left.S_{j(k-1)}(f), S_{j(k+1)}(f), \ldots, S_{j p}(f)\right],
\end{aligned}
$$

and $\boldsymbol{S}_{(\backslash j k)(\backslash j k)}^{-1}(f)$ is the inverse of the spectral matrix remaining when the $j$ th and $k$ th rows and columns of $\boldsymbol{S}(f)$ have been removed.

The partial coherence of $\left\{X_{j, t}\right\}$ and $\left\{X_{k, t}\right\}$ is defined as

$$
\gamma_{j k \mid(\backslash j k)}^{2}(f)=\frac{\left|S_{j k \mid(\backslash j k)}(f)\right|^{2}}{S_{j j \mid(\backslash j k)}(f) S_{k k \mid(\backslash j k)}(f)} .
$$

The method of calculating partial coherence using (2) with partial cross-spectra calculated via (1) will be called the 'direct method.'

Consider the case $p=4$ so that the order of the partial coherence is 2. Then, for example, (Bendat, 1978)

$$
S_{34 \mid 12}(f)=S_{34 \mid 1}(f)-S_{32 \mid 1}(f) S_{22 \mid 1}^{-1}(f) S_{24 \mid 1}(f)
$$

and

$$
\begin{aligned}
& S_{34 \mid 1}(f)=S_{34}(f)-S_{31}(f) S_{11}^{-1}(f) S_{14}(f) \\
& S_{32 \mid 1}(f)=S_{32}(f)-S_{31}(f) S_{11}^{-1}(f) S_{12}(f) \\
& S_{22 \mid 1}(f)=S_{22}(f)-S_{21}(f) S_{11}^{-1}(f) S_{12}(f) \\
& S_{24 \mid 1}(f)=S_{24}(f)-S_{21}(f) S_{11}^{-1}(f) S_{14}(f),
\end{aligned}
$$

so that $S_{34 \mid 12}(f)$ could be calculated directly in terms of ordinary cross-spectra. For $p=5$, i.e., order 3 ,

$$
S_{45 \mid 123}(f)=S_{45 \mid 12}(f)-S_{43 \mid 12}(f) S_{33 \mid 12}^{-1}(f) S_{35 \mid 12}(f),
$$

(e.g., Rosenberg et al. 1998) and each of the terms on the right could be written similarly to (3), and could then be expressed in terms of ordinary cross-spectra. The method of calculating partial coherence using (2) with partial cross-spectra calculated via this successive substitution method will be called the 'iterative method.'

An alternative computational approach which is wellsuited to large partial coherence orders makes use of the fact that

$$
\gamma_{j k \mid(\backslash j k)}^{2}(f)=\frac{\left|S^{j k}(f)\right|^{2}}{S^{j j}(f) S^{k k}(f)},
$$

where $S^{j k}(f)$ is the $(j, k)$ th term of the inverse spectral matrix $\boldsymbol{S}^{-1}(f)$. We note though that although (2) and (4) are equal, the numerators on the right on each side are not equal, in fact

$$
\left|S_{j k \mid(\backslash j k)}(f)\right|^{2}=\left|S^{j k}(f) /\left[S^{j j}(f) S^{k k}(f)-\left|S^{j k}(f)\right|^{2}\right]\right|^{2} .
$$

The method of calculating partial coherence using (4) will be called the 'inversion method.' Let $\Leftrightarrow$ denote 'if and only if.' $X_{j} \Perp X_{k} \mid X_{(\backslash j k)}$ means that $\left\{X_{j, t}\right\}$ and $\left\{X_{k, t}\right\}$ are uncorrelated given the other $(p-2)$ component processes. The following are all equivalent (Dahlhaus, 2000)

$$
\begin{aligned}
X_{j} \Perp X_{k} \mid X_{(\backslash j k)} & \Leftrightarrow \quad S_{j k \mid(\backslash j k)}(f)=0, \quad|f| \leq f_{\mathcal{N}} \\
& \Leftrightarrow \quad \gamma_{j k \mid(\backslash j k)}^{2}(f)=0, \quad|f| \leq f_{\mathcal{N}} \\
& \Leftrightarrow \quad S^{j k}(f)=0, \quad|f| \leq f_{\mathcal{N}} .
\end{aligned}
$$

\subsection{Computation of partial coherence}

As the order increases it is clear that the iterative method for partial coherence calculation rapidly becomes very complicated with many successive substitutions, and that when using estimated cross-spectra for actual calculations from real time series, estimation uncertainties will propagate in a way not currently understood.

The direct method for calculating partial coherencies is very time consuming since at each frequency and for the calculation of each of the $p(p-1) / 2$ partial coherencies, (1) requires the inversion of a $(p-2) \times(p-2)$ matrix.

For the inversion method for calculating partial coherencies at each frequency only a single inversion of a $p \times p$ matrix is required. As Eichler (1999) puts it, the inversion method "allows an efficient computation of all frequency 


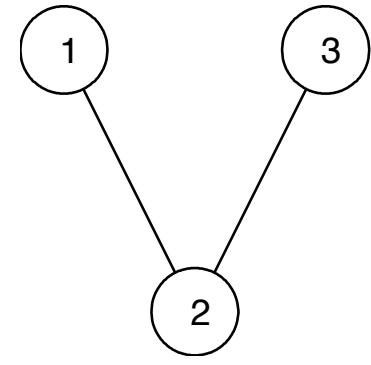

Figure 1: A simple undirected graph for which $1 \sim 2$ and $2 \sim 3$.

domain statistics..." This approach is similarly recommended in Dahlhaus (2000), Dahlhaus et al. (1997) and also used in Salvador et al. (2005), and it is used here.

The spectral matrix $\boldsymbol{S}(f)$ will be ill conditioned for inversion if the condition number (Strang, 1988)

$$
\kappa(f)=\frac{\lambda_{\max }(\boldsymbol{S}(f))}{\lambda_{\min }(\boldsymbol{S}(f))}
$$

is large, where $\lambda_{\max }(\boldsymbol{S}(f))$ and $\lambda_{\min }(\boldsymbol{S}(f))$ are the largest and smallest eigenvalues of $\boldsymbol{S}(f)$. The condition number will be relevant to the question of how best to compute (4) when cross-spectra are estimated, as is the case in practice.

\subsection{Partial coherence and conditional correlation graphs}

A graph $G=(V, E)$ consists of vertices $V$ and edges $E$, where $E \subset\{(j, k) \in V \times V: j \neq k\}$, i.e., edges connect pairs of distinct vertices. Our graphs are simple, there are neither loops from a vertex to itself nor any multiple edges between two vertices. Edges $(j, k) \in E$ for which both $(j, k) \in E$ and $(k, j) \in E$ are called undirected edges. In the visual representation of a graph a line joining $j$ to $k$ represents an undirected edge, signified by $j \sim k$. If all edges are undirected, the graph is said to be an undirected graph, which is assumed here. An example of a simple undirected graph is given in Fig. 1.

Let

$$
(j, k) \notin E \Leftrightarrow X_{j} \Perp X_{k} \mid X_{(\backslash j k)} .
$$

Then $G=(V, E)$ defines an undirected conditional correlation graph. (For Gaussian time series, a null partial correlation equates to independence between the $j$ th and $k$ th conditioned series, and in this case (8) defines a conditional independence graph.) So we see that an edge is missing from the graph if $\left\{X_{j, t}\right\}$ and $\left\{X_{k, t}\right\}$ are uncorrelated given the other $(p-2)$ component processes. Putting (5), (6) and (8) together we obtain

$$
(j, k) \notin E \Leftrightarrow S^{j k}(f)=0 \Leftrightarrow \gamma_{j k \mid(\backslash j k)}^{2}(f)=0, \quad|f| \leq f_{\mathcal{N}} .
$$

So missing edges in the conditional independence graph are identifiable from (i) zeros in the inverse spectral matrix, or (ii) zero partial coherences, for $|f| \leq f_{\mathcal{N}}$. Hence to determine the form of the graph - basically the presence or absence of edges — we need to test (9). Also, we shall be interested in both the inverse spectral matrix and partial coherence, since null values of these have the same implication for the absence of an edge.

Note that there are $p(p-1) / 2$ possible interactions between the series, or equivalently, edges to the graph. This is simply the number of off-diagonal terms in the upper (or lower) triangle of the spectral matrix.

\subsection{Estimation of the spectral matrix}

In practice $\boldsymbol{S}(f)$ will not be known and it will have to be estimated, as $\hat{\boldsymbol{S}}(f)$ say, from measured time series. A widely-used current spectral estimation method is multitaper spectral analysis (e.g., Percival \& Walden, 1993). We make use of a set of $K$ real-valued orthonormal taper sequences $\left\{h_{k, t}, t=0, \ldots, N-1\right\}$, for $k=0, \ldots, K-1$. Since they are orthonormal we know $\sum_{t} h_{j, t} h_{k, t}=1$ if $j=k$, and zero otherwise.

We form the product $h_{k, t} \boldsymbol{X}_{t}$ of the $t$ th component of the $k$ th taper with the $t$ th component of the vector-valued process, and then compute the (scaled) vector Fourier transform

$$
\boldsymbol{J}_{k}(f) \equiv(\Delta t)^{1 / 2} \sum_{t=0}^{N-1} h_{k, t} \boldsymbol{X}_{t} \mathrm{e}^{-\mathrm{i} 2 \pi f t \Delta t} .
$$

The estimator of the $p \times p$ spectral matrix $\boldsymbol{S}(f)$ is given by

$$
\hat{\boldsymbol{S}}(f)=\frac{1}{K} \sum_{k=0}^{K-1} \boldsymbol{J}_{k}(f) \boldsymbol{J}_{k}^{H}(f),
$$

where ${ }^{H}$ denotes transpose and complex conjugation. Then $\hat{S}_{l m}(f)$ can be written

$$
\frac{\Delta t}{K} \sum_{k=0}^{K-1}\left\{\sum_{s=0}^{N-1} h_{k, s} X_{l, s} \mathrm{e}^{-\mathrm{i} 2 \pi f s \Delta t}\right\}\left\{\sum_{t=0}^{N-1} h_{k, t} X_{m, t} \mathrm{e}^{\mathrm{i} 2 \pi f t \Delta t}\right\} .
$$

Provided $K \geq p$, then for large $N$, (e.g., Walden, 2000)

$$
\hat{\boldsymbol{S}}(f) \stackrel{\mathrm{d}}{=} \begin{cases}(1 / K) \mathcal{W}_{p}^{C}\{K, \boldsymbol{S}(f)\}, & \text { for }|f| \neq 0, f_{\mathcal{N}} \\ (1 / K) \mathcal{W}_{p}\{K, \boldsymbol{S}(f)\}, & \text { for }|f|=0, f_{\mathcal{N}}\end{cases}
$$

where $\mathcal{W}_{p}^{C}\{K, \boldsymbol{S}(f)\}$ denotes the $p$-dimensional complex Wishart distribution with $K$ degrees of freedom and mean $K \boldsymbol{S}(f)$, and $\mathcal{W}_{p}\{K, \boldsymbol{S}(f)\}$ denotes the real equivalent. The distributional result (10) enables some useful results to be derived for statistics derived from $\hat{\boldsymbol{S}}(f)$, such as the partial coherence estimator $\hat{\gamma}_{j k \mid(\backslash j k)}^{2}(f)$.

\section{Data analysis}

\subsection{Subjects}

The techniques to be discussed in this paper are applied to EEG recordings from 8 patients diagnosed by a psychiatrist as having paranoid schizophrenia by DSM-IV criteria. The patients were recruited from three acute psychiatric 


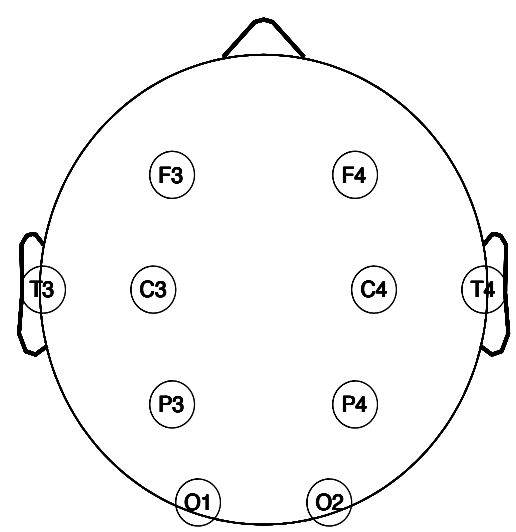

Figure 2: Positions of 10 electrodes

\begin{tabular}{|c|c|c|c|c|c|c|c|c|c|}
\hline 1 & 2 & 3 & 4 & 5 & 6 & 7 & 8 & 9 & 10 \\
\hline F3 & F4 & C3 & C4 & P3 & P4 & O1 & O2 & T3 & T4 \\
\hline
\end{tabular}

Table 1: Process numbers corresponding to electrode positions.

wards of Charing Cross Hospital, London. All patients were on antipsychotic medication and were clinically stable. EEG recordings were also made for 16 healthy male volunteers (controls) of comparable age. All subjects gave written informed consent. Ethical approval came from the Riverside Research Ethics committee.

\subsection{EEG data and initial treatment}

EEG was recorded from scalp sites with reference during recording to the left ear. The results reported herein concern data from 10 electrode sites, namely F3, F4, C3, C4, P3, P4, O1, O2, T3, T4, illustrated in Fig.2 and plotted using EEGLAB software, (Delorme and Makeig, 2004). The time series from these electrodes form the $p=10 \mathrm{com}$ ponent processes of $\left\{\boldsymbol{X}_{t}\right\}$ and were re-labelled as component processes 1-10, respectively, as in Table 1. The data were recorded with signal bandpass of $0.1-100 \mathrm{~Hz}$, and downsampled in the absence of aliasing to a sample interval $\Delta t=4 \mathrm{~ms}$ giving a Nyquist frequency of $f_{\mathcal{N}}=125 \mathrm{~Hz}$. The epochs used here are for resting conditions (eyes closed). Epochs containing EEG values outside the range $\pm 100 \mu \mathrm{V}$ were excluded. As a result, 30 epochs were available for analysis for the 10 electrodes for all 8 patients and 10 of the 16 controls. Each epoch contained $N=512$ time series values. The series were standardized to have a unity sample variance and 'mean-corrected' spectral estimators were used - see for example Percival \& Walden (1993, p. 500).

\subsection{Resulting spectral matrices}

We have two sets of individuals, patients and controls, for each set we have recordings from different electrode sites, and for each of these we have recordings for different

\begin{tabular}{|c|c|c|c|c|c|c|c|c|c|}
\hline & F4 & C3 & $\mathrm{C} 4$ & P3 & $\mathrm{P} 4$ & $\overline{\mathrm{O} 1}$ & $\overline{\mathrm{O} 2}$ & $\mathrm{~T} 3$ & $\mathrm{~T} 4$ \\
\hline F3 & 1 & 2 & 3 & 4 & 5 & 6 & 7 & 8 & 9 \\
\hline $\mathrm{F}$ & & 10 & 11 & 12 & 13 & 14 & 15 & 16 & 17 \\
\hline & C3 & & 18 & 19 & 20 & 21 & 22 & 23 & 24 \\
\hline \multicolumn{4}{|c|}{ C4 } & 25 & 26 & 27 & 28 & 29 & 30 \\
\hline \multicolumn{5}{|c|}{ P3 } & 31 & 32 & 33 & 34 & 35 \\
\hline \multirow{2}{*}{\multicolumn{7}{|c|}{$\frac{\mathrm{P} 4}{\mathrm{O} 1}$}} & 37 & 38 & 39 \\
\hline & & & & & & & 40 & 41 & 42 \\
\hline \multicolumn{8}{|c|}{$\mathrm{O} 2$} & 43 & 44 \\
\hline \multicolumn{9}{|c|}{ T3 } & 45 \\
\hline
\end{tabular}

Table 2: Interaction indices used to identify possible interactions between series recorded at specified electrode locations.

epochs. We denote the $p \times p=10 \times 10$ spectral matrix for patient $a$ and epoch $b$ by $\hat{\boldsymbol{S}}(f ; P, a, b)$, where $a=1, \ldots, 8$ and $b=1, \ldots, 30$; likewise for control $a$ and epoch $b$ by $\hat{\boldsymbol{S}}(f ; C, a, b)$, where $a=1, \ldots, 10$ and $b=1, \ldots, 30$. For a generic case, where the extra labels are not important, we shall just use the simpler notation $\hat{\boldsymbol{S}}(f)$.

Spectral matrices were computed using $K=20$ sine tapers giving an effective estimation bandwidth of $B=$ $(K+1) /[(N+1) \Delta t] \approx 10.3 \mathrm{~Hz}$ (see e.g., Walden et al., 1995). The choice of $K=20$ was determined by looking at spectra and cross-spectra for a number of patients and controls and finding a $K$ such that the resulting estimates are neither too smooth nor too erratic, and important detail is preserved. This procedure is known as 'window closing' (e.g., Percival \& Walden, 1993, p. 276).

Note the number of possible interactions or edges in the graph corresponds to the number of off-diagonal terms of our spectral matrices, i.e., there are $p(p-1) / 2=45$ possible interactions for our $p=10$ electrodes. The numerical labelling of these possible interactions via interaction indices is given in Table 2 .

\subsection{Removal of interference}

So-called 'power line pick-up' is a common problem in EEG spectral analysis. It arises when the EEG electrodes pick-up interference from the operational power supply frequency $(50 \mathrm{~Hz}$ in Europe). Inaccurate amplifier cancellation leaves a residual signal at this frequency which takes the form of a line in the spectrum at $50 \mathrm{~Hz}$; however because of the effective resolution bandwidth of the spectral estimator, this appears as a near-rectangular block centred at $50 \mathrm{~Hz}$. Using techniques described in detail in Percival and Walden (1993, Chapter 10) the line can be detected, estimated and removed and the spectrum appropriately reshaped. Fig. 3 shows the estimated power spectrum $\hat{S}(f ; C, 1,1)$, for electrode $\mathrm{F} 3$, and corresponding autocovariance sequence, before and after removal of the $50 \mathrm{~Hz}$ line and spectral reshaping. The autocovariance sequence in Fig. 3(b) displays the strong $50 \mathrm{~Hz}$ ripple which is absent after line removal in Fig. 3(d).

Best reshaping results are achieved when $50 \mathrm{~Hz}$ coincides with an element of the frequency grid. We hence 

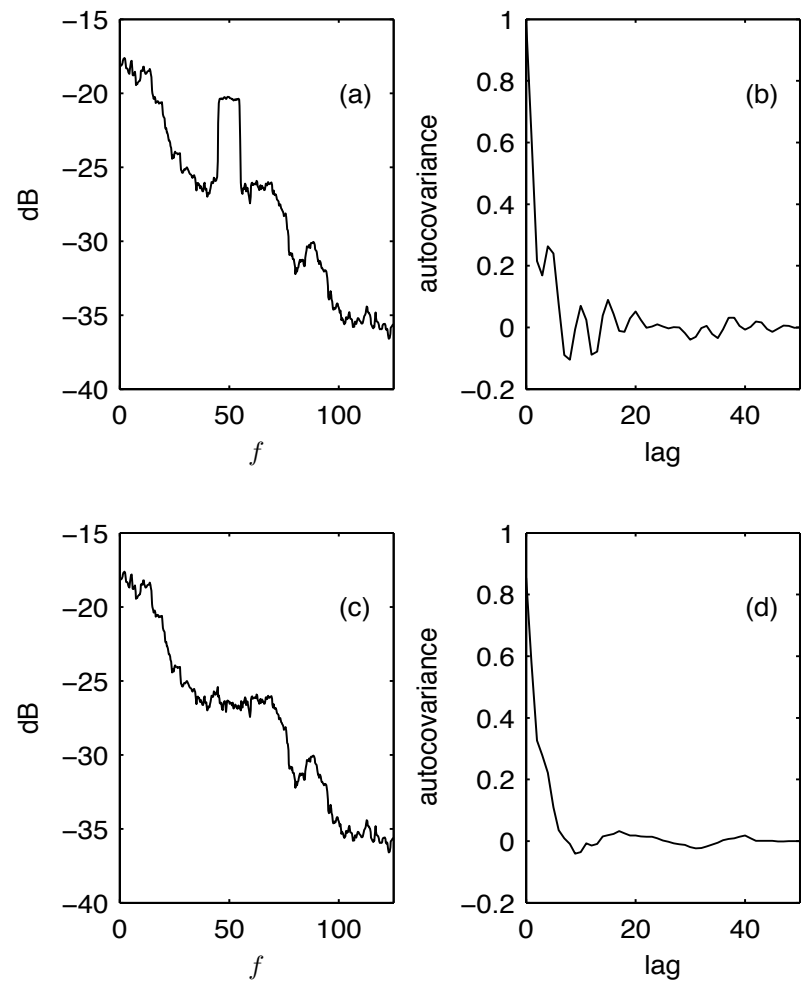

Figure 3: (a) and (b), show respectively, the estimated power spectrum $\hat{S}(f ; C, 1,1)$, for electrode $\mathrm{F} 3$, and corresponding autocovariance sequence. (c) and (d) show the same after removal of the $50 \mathrm{~Hz}$ line and spectral reshaping.

chose to sample at 501 points in $[0,125] \mathrm{Hz}$, so that $\Delta f=$ $125 / 500=0.25 \mathrm{~Hz}$. The Fourier transform length used was thus 1000 rather than a more typical 1024 .

The same scheme can correct other isolated line interferences, which were found to occur in a few of the time series.

\subsection{Unbiasing of partial coherence estimator}

The basic raw estimator of the partial coherence follows from (2) as

$$
\hat{\gamma}_{j k \mid(\backslash j k)}^{2}(f)=\frac{\left|\hat{S}_{j k \mid(\backslash j k)}(f)\right|^{2}}{\hat{S}_{j j \mid(\backslash j k)}(f) \hat{S}_{k k \mid(\backslash j k)}(f)},
$$

which corresponds to replacing the spectral components by their estimators. This quantity can be debiased by using instead

$$
\check{\gamma}_{j k \mid(\backslash j k)}^{2}(f)=\frac{\hat{\gamma}_{j k \mid(\backslash j k)}^{2}(f)-\frac{1}{n-p+2}}{1-\frac{1}{n-p+2}},
$$

where $n$ is the number of complex degrees of freedom of the spectral estimator. For a single individual and epoch $n=K$, so that $n-p+2=12$, and the bias correction will thus be small.

\section{Stabilising the spectral matrix inversion}

\subsection{The problems}

It was pointed out that (10) holds for $K \geq p$. In fact if $K<p$, then $\hat{\boldsymbol{S}}(f)$ is always singular. In the discussion below we assume $K \geq p$, i.e., the number of orthonormal tapers used, (equivalent to the number of complex degrees of freedom), is at least as large as the number of channels of data.

The two main issues affecting spectral matrix inversion are (i) spectral nulls induced by linear correlation, an effect due to the nature of the data, and (ii) spectral side-lobe leakage, caused by imperfect spectral estimation.

Consider the case of just three series $(p=3)$ for illustration. If $X_{1, t}$ and $X_{3, t}$ are perfectly linearly related at frequency $f$, then the spectral matrix $\boldsymbol{S}(f)$ will be singular and $S_{11 \mid 3}(f)=0$. As this is a denominator term in $\gamma_{12 \mid 3}^{2}(f)$ in (2) this will cause serious problems. Such spectral nulls can occur at isolated frequencies, or over frequency bands. When using estimated spectra in practice the estimated spectral matrix can be rendered less nearsingular by schemes such as matrix diagonal up-weighting aimed at increasing the values of the smallest eigenvalues (e.g., Carlson, 1988).

Spurious values can also occur in the estimated spectral matrix $\hat{\boldsymbol{S}}(f)$ due to spectral side-lobe leakage from adjacent frequencies. To deal with such problems we consider the use of diagonal up-weighting. This is also equivalent to adding white noise to the time series, but is designed so that the added noise dominates side-lobe leakage, typically at the cost of some very small reduction in estimated partial coherencies.

Diagonal up-weighting thus simultaneously addresses both spectral matrix inversion instability and side-lobe leakage effects, and is thus more potentially useful than singular-value decomposition which would only help with the first problem.

We now discuss diagonal up-weighting in more detail.

\subsection{Matrix diagonal up-weighting}

The matrix diagonal up-weighting scheme we shall use consists of the following steps:

- Evaluate the maximum value, over the chosen frequency range, of each diagonal element of $\hat{\boldsymbol{S}}(f)$. For a $p \times p$ spectral matrix, compute $a_{i}=\max _{f}\left(\hat{S}_{i i}(f)\right)$, where $i=1, \ldots, p$ and arrange the values $a_{i}$ in a diagonal matrix and call it $\boldsymbol{A}$.

- Multiply by a fixed up-weighting parameter $\zeta$ to get $\boldsymbol{B}=\zeta \boldsymbol{A}$.

- Finally, add the matrix $\boldsymbol{B}$ to the original spectral matrix to obtain $\hat{\boldsymbol{S}}(f)+\boldsymbol{B}$, which becomes the new estimated spectral matrix $\hat{\boldsymbol{S}}(f)$. 

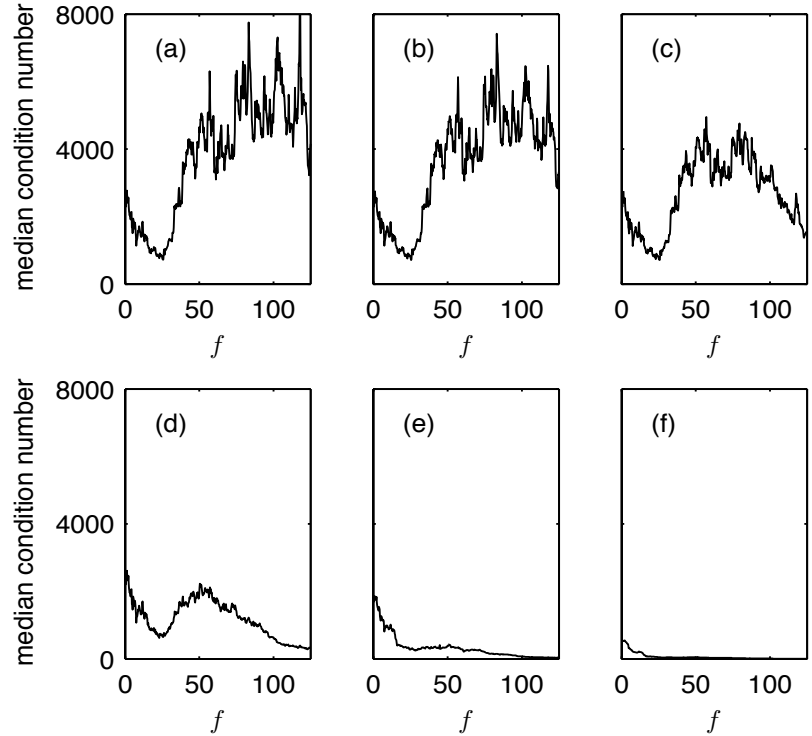

Figure 4: Plots comparing the median condition number over the controls as a function of frequency for values of $\zeta$ of : (a) 0 , (b) $10^{-6}$, (c) $10^{-5}$, (d) $10^{-4}$, (e) $10^{-3}$, and (f) $10^{-2}$. Here $K=20$ and epoch $b=1$.

The up-weighting of the diagonal elements is nonuniform, and in this sense has elements in common with generalised ridge regression (de Boer, 2005).

The first important practical issue is to appreciate the effect on estimated partial coherence of changing the parameter $\zeta$. Results in sections 4.3 and 4.4 are for the representative first epoch.

\subsection{The up-weighting parameter $\zeta$}

We illustrate the effect of $\zeta$ in Fig. 4 which shows the median (estimated) condition number $\hat{\kappa}(f)$ over controls as a function of frequency using the diagonal up-weighting scheme, for $\zeta$ varying between 0 and 0.01 . As expected, the condition number falls from very large values to moderate values as $\zeta$ increases, indicating increasingly stable invertibility of the estimated spectral matrix with increasing $\zeta$. A similar dramatic effect is also seen for patients. It is important to appreciate that the ill-conditioning for $\zeta=0$, the basic estimated spectral matrix, is present for frequencies within bands with strong signal (e.g., alpha, beta) - it does not just occur where signal is weak - and is hence a fundamental problem which must be addressed.

To demonstrate the effect on spectral leakage of the upweighting, we band-pass filtered the recorded series to the beta frequency range of $[12,30] \mathrm{Hz}$ using a Butterworth filter of length 125 and order 5. Fig. 5 shows the debiased estimated partial coherences $\check{\gamma}_{35 \mid(\backslash 35)}^{2}(f)$ for control 1 for a range of $\zeta$ values. We see that without up-weighting, $(\zeta=0)$, the partial coherence is large for frequencies from zero to Nyquist, even though the time series were bandpass filtered to the beta range. This is caused by small amounts of power leaking from high power to low power
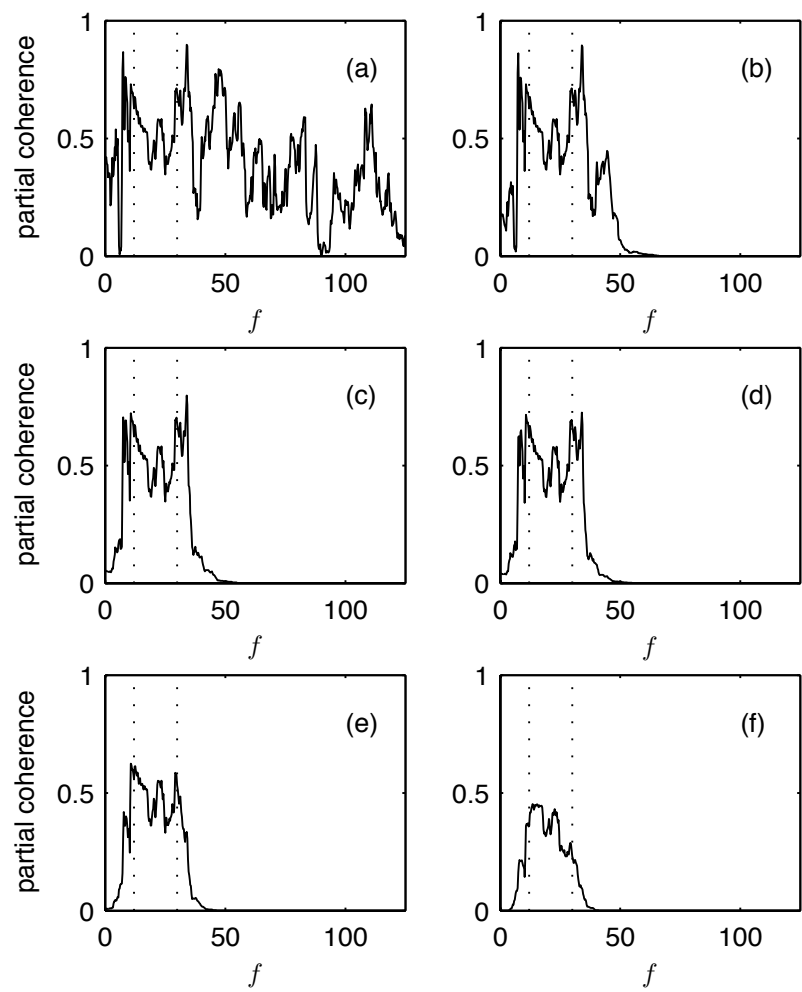

Figure 5: Debiased estimated partial coherences for control 1 and electrodes C3 and P3 for $\zeta$ values of (a) 0 , (b) $10^{-6}$, (c) $5 \cdot 10^{-5}$, (d) $10^{-4}$, (e) $10^{-3}$ and (f) $10^{-2}$. The vertical dotted lines delineate the beta pass-band. Here $K=20$ and epoch $b=1$.

regions of the spectra, an inevitable part of spectral estimation (e.g., Percival \& Walden, 1993), for example giving rise to small non-zero denominator terms in (2), which then inflate the small numerator. Contrariwise, we see that when $\zeta=5 \cdot 10^{-5}$ or $10^{-4}$, the partial coherence is confined to a band of frequencies much closer to the nominal pass-band, and the partial coherence values within the pass-band are virtually unchanged. For $\zeta=10^{-3}$, the partial coherence is confined even more closely to a band of frequencies approximating the nominal pass-band, but the partial coherence values within the pass-band are more noticeably modified compared to when $\zeta=0$. This modification is even more noticeable when $\zeta=10^{-2}$ - any useful information in the pass-band has been destroyed.

The reported behaviour is typical of what we encountered - increasing $\zeta$ increases the stability of matrix inversion but after a certain point useful information is destroyed via a serious deformation of the partial coherence. The next step is to determine how to choose an 'optimum' value for $\zeta$ by a trade-off of these two effects. 


\subsection{Choosing $\zeta$}

To choose a suitable value for $\zeta$ we propose a graphical approach based on the measure defined as

$$
\varphi_{j k}=-\frac{1}{2 f_{\mathcal{N}}} \int_{-f_{\mathcal{N}}}^{f_{\mathcal{N}}} \log \left[1-\gamma_{j k \mid(\backslash j k)}^{2}(f)\right] \mathrm{d} f .
$$

If $\gamma_{j k \mid(\backslash j k)}^{2}(f)=0$ everywhere, then $\varphi_{j k}=0$ and if $\gamma_{j k \mid(\backslash j k)}^{2}(f)=1$ everywhere, then $\varphi_{j k}=\infty$. If the time series are Gaussian then this corresponds to the partial mutual information (PMI) (Granger and Hatanaka, 1964). We make use of this measure as an objective function without claiming Gaussianity - in the same way Brillinger (1992) did when using mutual information but for convenience of nomenclature we shall still call it the PMI.

Consider again band-pass filtering the recorded series to the beta frequency range of $[12,30] \mathrm{Hz}$. Then we estimate the contribution to the PMI (by numerical integration of the partial coherence) within and outside the pass-band, replacing the true unknown partial coherence in (13) by the debiased estimated coherence (dependent on $\zeta)$; we call these two quantities the in-band PMI, $\varphi_{j k}^{(I)}(\zeta)$ and the out-of-band PMI, $\varphi_{j k}^{(O)}(\zeta)$. This was done for a range of $\zeta$ values, and finally the two PMIs were standardized by dividing by their respective maximum values over $\zeta$ to give the in-band standardized PMI, $\varphi_{j k}^{(I)}(\zeta) / \max _{\zeta}\left\{\varphi_{j k}^{(I)}(\zeta)\right\}$ and the out-of-band standardized PMI, $\varphi_{j k}^{(O)}(\zeta) / \max _{\zeta}\left\{\varphi_{j k}^{(O)}(\zeta)\right\}$. These two quantities are calculated for all possible interactions $j, k$ or edges $j \sim k$, and the medians found, and these are then averaged over individuals to give the plots in Fig. 6 for patients and controls. Thinking in terms of Fig. 5, we want to keep the in-band standardized PMI close to 1, (i.e., close to the value for $\zeta=0$,) while making the out-of-band standardized PMI as small as possible. We can see from Fig. 6 that, for both patients and controls, this can be best achieved as the plot curve starts to bend away from horizontal towards vertical, the 'knee' point, corresponding to a $\zeta$ value of $5 \cdot 10^{-5}$ or $10^{-4}$, (the 5 th and 6th points starting from the top right corner, marked by a ' + ' and ' $*$ ' respectively). Other frequency bands including $[4,8],[8,12],[4,30],[0,60]$, $[12,60]$ and $[30,60] \mathrm{Hz}$ all resulted in the choice of a $\zeta$ value between $10^{-5}$ and $10^{-4}$, and the latter was chosen for all subsequent analysis.

\subsection{Partial coherencies}

Using the values of the analysis parameters above, namely $K=20, \zeta=10^{-4}$, partial coherencies were estimated for patients and controls. An example set are shown in Fig. 7. These have been debiased according to (12). As expected, partial coherencies are generally higher at lower frequencies, but interestingly the F3-F4 connection gives rise to a large partial coherence across the full frequency range, a persistent effect across controls, patients and epochs.
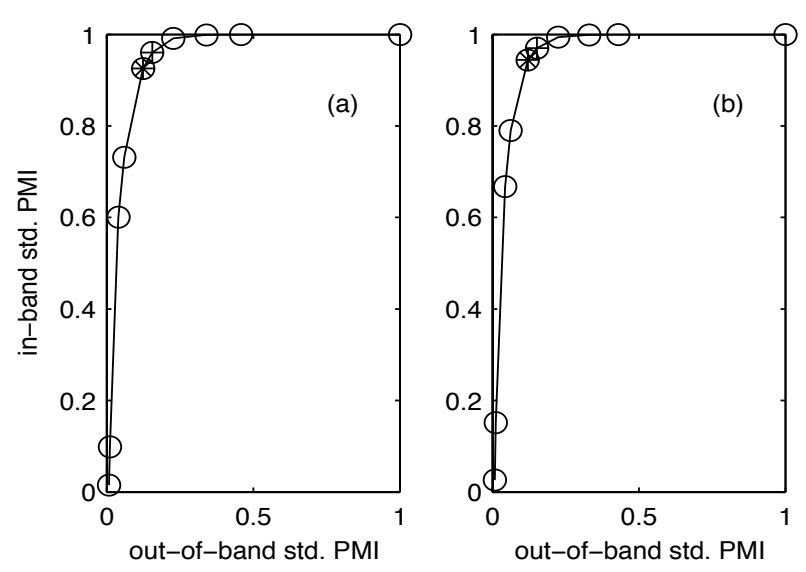

Figure 6: In-band standardized PMI versus out-of-band standardized PMI for (a) patients, and (b) controls. Starting in the top-right corner of each plot, the circles are for $\zeta=0,10^{-7}, 10^{-6}, 10^{-5}, 5$. $10^{-5}, 10^{-4}, 5 \cdot 10^{-4}, 10^{-3}, 10^{-2}, 10^{-1}$. Here $K=20$ and epoch $b=1$.

\section{Hypothesis testing}

\subsection{The problem}

To determine the absence of edges in our graphical model we need, from (9), to test for the condition

$$
\gamma_{j k \mid(\backslash j k)}^{2}(f)=0, \quad|f| \leq f_{\mathcal{N}} .
$$

As pointed out in Dahlhaus et al. (1997, p. 101) it is impractical to try to conduct this test at all frequencies $|f| \leq f_{\mathcal{N}}$. We therefore consider testing the hypotheses

$$
H_{l}: r_{j k}\left(f_{l}\right)=0, \text { for all } l=1, \ldots, L,
$$

where for notational simplicity we have set $r_{j k}(f) \equiv$ $\gamma_{j k \mid(\backslash j k)}^{2}(f)$, and $f_{1}, \ldots, f_{L}$ are a set of frequencies in the range $\left[0, f_{\mathcal{N}}\right]$, to be defined later. (By symmetry we need only consider positive frequencies.) This is a multiple hypothesis testing problem.

\subsection{Multiple hypothesis testing}

If each hypothesis is separately tested at a level $\alpha$ then the probability of one or more false rejections rapidly increases with $L$. For independent test statistics and $\alpha=$ 0.05, Lehmann \& Romano (2005, p. 349) show that the probability of at least one false rejection rises from 0.05 for $L=1$ to 0.4 for $L=10$. A suitable approach is instead to require that the probability of one or more false rejections should not exceed a certain level. This probability is known as the family-wise error rate (FWER). In this paper we consider multiple testing procedures for which FWER $\leq \alpha$.

Lehmann \& Romano (2005, p. 350) point out that the well-known Bonferroni method satisfies this requirement, but the ability to detect cases in which the hypothesis is false will typically be very low since all hypotheses are tested at the same $\alpha / L$ level. It is desirable to increase 

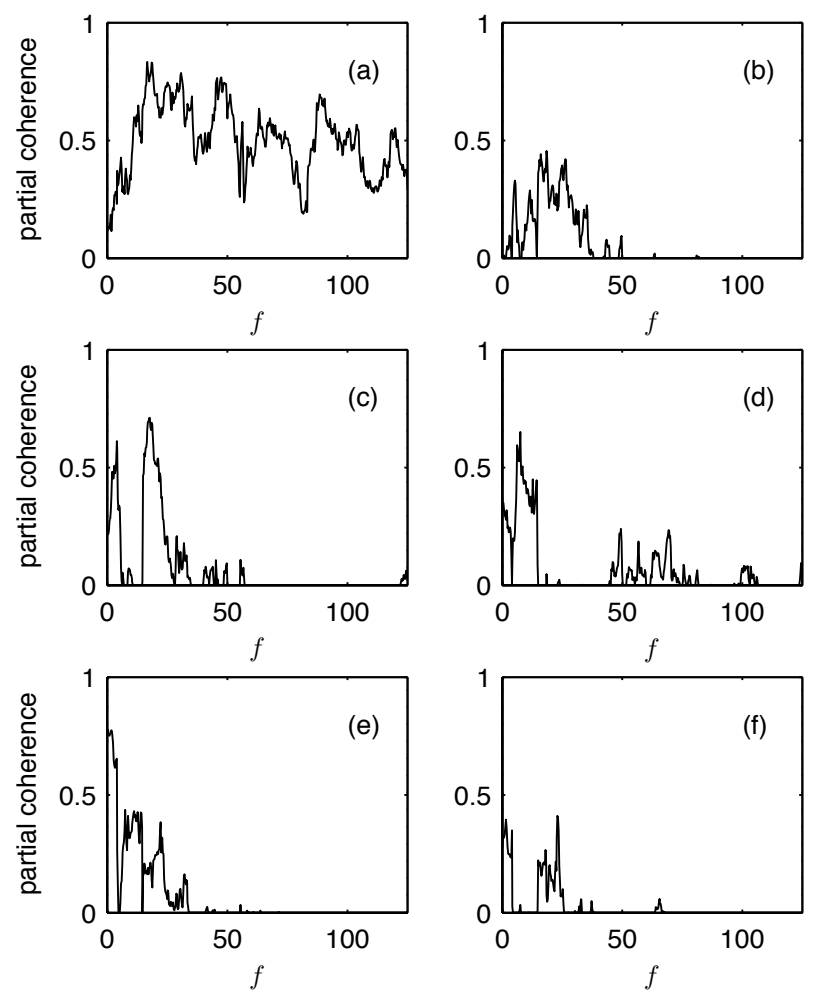

d)

Figure 7: Debiased partial coherencies for a patient, epoch 1. Interactions are (a) F3-F4, (b) F4-C3, (c) C3-C4, (d) C4-T4, (e) P3-P4, and (f) P3-O2.

the levels of the tests over $\alpha / L$ without an increase in the FWER. We thus concentrate our attention on stepdown procedures.

\subsection{The maximin stepdown procedure}

Multiple hypothesis testing may be addressed via the maximin stepdown procedure (Lehmann \& Romano, 2005, section 9.2). For notational simplicity set $R_{j k}(f) \equiv$ $\hat{\gamma}_{j k \mid(\backslash j k)}^{2}(f)$. We wish to test the hypotheses (14) against general alternatives. For a given $(j, k)$ define $R_{l} \equiv$ $\hat{R}_{j k}\left(f_{l}\right), l=1, \ldots, L$. We use the partial coherence estimators $R_{1}, \ldots, R_{L}$ as our test statistics for the procedure. Denote the ordered $R$-values by $R_{(1)} \leq R_{(2)} \leq \ldots \leq R_{(L)}$ and the corresponding hypotheses by $H_{(1)}, \ldots, H_{(L)}$.

- Step 1: if $R_{(L)}<C_{1}$, accept $H_{1}, \ldots, H_{L}$.

- Step 2: if $R_{(L)} \geq C_{1}$ but $R_{(L-1)}<C_{2}$, reject $H_{(L)}$ and accept $H_{(1)}, \ldots, H_{(L-1)}$.

- Step l: if $R_{(L)} \geq C_{1}, \ldots, R_{(L-l+2)} \geq C_{l-1}$, but $R_{(L-l+1)}<C_{l}$ reject $H_{(L)}, \ldots, H_{(L-l+2)}$ and accept $H_{(1)}, \ldots, H_{(L-l+1)}$.
- Step $L+1$ : if $R_{(L)} \geq C_{1}, \ldots, R_{(1)} \geq C_{L}$, reject the hypotheses $H_{(1)}, \ldots, H_{(L)}$.

The critical values $C_{l}$ are determined by

$$
G_{L-l+1}\left(C_{l}\right)=1-\alpha
$$

where

$$
G_{j}(c)=P\left(\max \left(R_{1}, \ldots, R_{j}\right)<c \mid H_{1}, \ldots, H_{j}\right),
$$

is the distribution function of the largest of $R_{1}, \ldots, R_{j}$ given that $H_{1}, \ldots, H_{j}$ are true.

The maximin procedure can be expressed in an alternative way. Denote the $p$-value of the statistic $R_{l}$ by $P_{l}$, and denote the ordered $p$-values by $P_{(1)} \leq P_{(2)} \leq \ldots \leq P_{(L)}$. If $F_{R}(\cdot)$ denotes the common (marginal) distribution of $R_{l}$ under the null hypothesis, i.e., $P_{l}=1-F_{R}\left(R_{l}\right)$, then

$$
P_{(l)}=1-F_{R}\left(R_{(L-l+1)}\right) \quad \text { for } l=1, \ldots, L .
$$

The rejection criteria $R_{(L)} \geq C_{1}, R_{(L-1)} \geq C_{2}, \ldots$ of the maximin procedure become

$$
P_{(1)} \leq \alpha_{1}, P_{(2)} \leq \alpha_{2}, \ldots
$$

Lehmann \& Romano (2005, Theorem 9.2.4) prove that

$$
\begin{aligned}
1-\alpha_{l} & =F_{R}\left(G_{L-l+1}^{-1}(1-\alpha)\right) \\
\frac{\alpha}{L-l+1} & \leq \alpha_{l} \leq \alpha
\end{aligned}
$$

Our outstanding problem at this point is the determination of the critical values $\left\{C_{l}\right\}$ defined by (15).

\subsection{Independent partial coherencies}

Here we consider the case when the partial coherence estimators at frequencies $\left\{f_{l}\right\}$ are independent.

Under the assumption of independence of $R_{1}, \ldots, R_{L}$ we have that

$$
G_{L-l+1}\left(C_{l}\right)=F_{R}^{L-l+1}\left(C_{l}\right)
$$

where $F_{R}(\cdot)$ is the common distribution function of each $R_{l}$. Thus,

$$
C_{l}=F_{R}^{-1}\left((1-\alpha)^{\frac{1}{L-l+1}}\right) .
$$

Under (10) and the null hypothesis of zero partial coherence, the partial coherence estimator, $R_{l}$, estimated with $n$ complex degrees of freedom, has a beta distribution with parameters 1 and $n-p+1$ with distribution function

$$
F_{R}(c)=1-(1-c)^{n-p+1}
$$

Hence

$$
C_{l}=1-\left(1-(1-\alpha)^{\frac{1}{L-l+1}}\right)^{\frac{1}{n-p+1}}
$$

It is important to realise that the beta distribution result used here applies to the basic raw estimator of the partial coherence (11), not that after debiasing, (12). 


\subsection{Dependent partial coherencies}

To find the distribution function $G_{j}(c)$ in the case of correlated partial coherent estimators is possible only via extensive computations and further approximations and assumptions. Instead we can adopt the testing approach due to Holm (1979) which is a special case of the stepdown procedures. Rather than adjusting the significance levels at each step as in (17), $\alpha_{l}$ is set to the lower bound of $\alpha /(L-l+1)$ in (18). As a result the Holm approach is more conservative (accepts the null hypothesis more readily) than the maximin procedure.

At each level the critical value can now be evaluated simply using

$$
C_{l}=F_{R}^{-1}\left(1-\alpha_{l}\right)=1-\left(\frac{\alpha}{L-l+1}\right)^{n-p+1},
$$

so that the Holm method is very practical for the case of dependent partial coherencies.

For a single individual and epoch, $n=K$ in (19) and (20).

\subsection{Using the stepdown procedure}

Consider the stepdown procedure. If $R_{(L)}<C_{1}$, we accept $H_{1}, \ldots, H_{L}$, in which case we would conclude $(j, k) \notin$ $E$, i.e., there is no edge in our graphical model between series $j$ and $k$. If $R_{(L)} \geq C_{1}$ we reject $H_{(L)}$, and so our test would say to put an edge in the graphical model since there is an interaction between series $j$ and $k$.

However, by choosing to continue with the algorithm we can investigate the strength of the interaction. If the procedure stops at the $(l+1)$ th step then $l$ hypotheses for zero partial coherence are rejected. We could then consider the ratio of the number of rejected hypothesis to the total number of hypothesis investigated, namely,

$$
R R H=l / L,
$$

(where $R R H$ stands for the ratio of rejected hypotheses), as a measure of the strength of the interaction between series $j$ and $k$.

\subsection{Choice of testing frequencies}

The frequency grid used in our computations was discussed in section 3.4. The increment was $\Delta f=f_{l+1}-f_{l}=$ $0.25 \mathrm{~Hz}$.

In section 3.3 the effective bandwidth when using $K$ sine tapers was defined and denoted by $B$. Thus if $\left|f_{l}-f_{m}\right|>B$, we can consider $R_{j k}\left(f_{l}\right)$ and $R_{j k}\left(f_{m}\right)$ to be independent for any $f_{l}, f_{m} \in\left[0, f_{\mathcal{N}}\right]$. Hence by using frequencies in the range 0 to $f_{\mathcal{N}}$ spaced apart by at least $B$ we can conduct hypothesis testing under the assumption of independent partial coherencies. For the full frequency range $[0,125] \mathrm{Hz}$ and $B=10.3 \mathrm{~Hz}$ as in section 3.3 we would thus have $L=13$ frequencies at which to define the hypothesis tests. However if we restrict our frequency range to the beta band we would have $L=2$ while $L$ would be unity for the
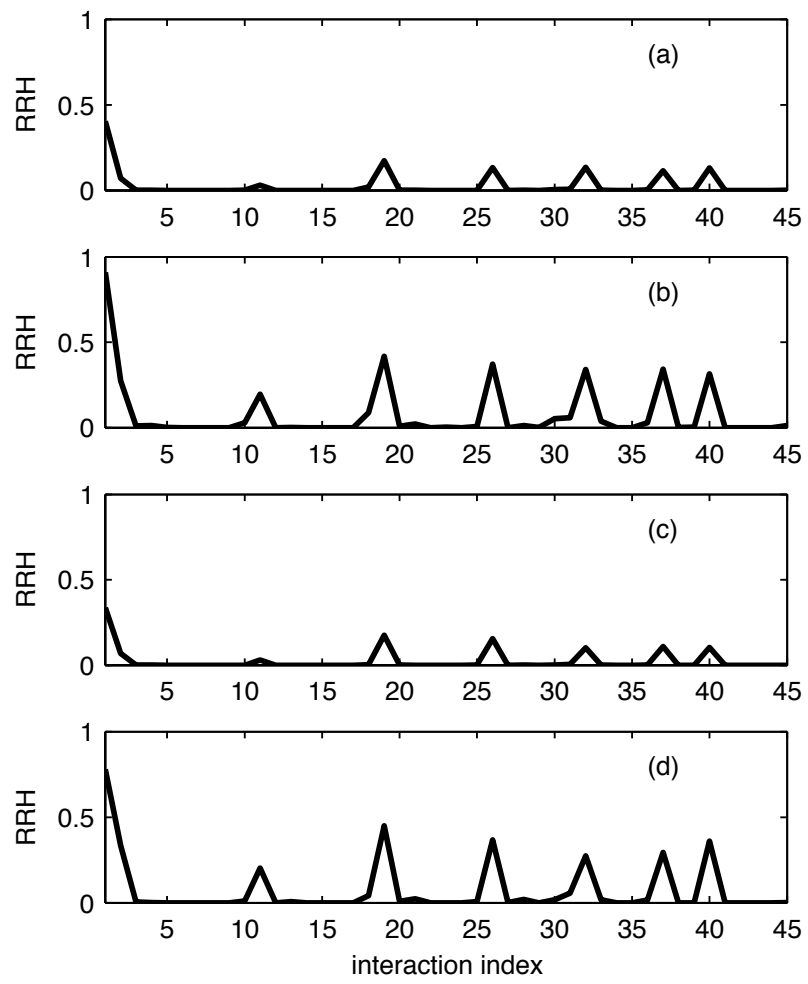

Figure 8: The median over individuals followed by the median over epochs of the $R R H$, by interaction index, for (a) patients and Holm scheme, (b) patients and independent scheme, (c) controls and Holm scheme, and (d) controls and independent scheme. Here $K=20$, and $\alpha=0.05$.

delta, theta and alpha bands. In these cases the critical values for the stepdown procedure are specified by (19).

If we use all the frequencies $f_{l}$ between zero and $f_{\mathcal{N}}$, (or within any specified band, alpha, beta etc), then our hypothesis testing makes use of dependent partial coherencies. The critical values for the stepdown procedure are then specified by $(20)$.

\subsection{Comparison of independent and dependent schemes}

As has been explained, the independent testing method is hampered by the fact that the number of tests which can be performed can become extremely limited for narrow ranges of frequencies. On the other hand, the Holm procedure, while allowing testing at all frequencies, is more conservative. Let us compare the two approaches for the full-band case $f \in\left[0, f_{\mathcal{N}}\right]$.

Fig. 8 plots the 'averaged' $R R H$, (over individuals and epochs) for patients and controls. We denote this quantity by $\overline{R R H}$. The interaction indices are as in Table 2. The Holm procedure produces 'weaker' interactions, measured by the averaged $R R H$, compared to the independent alternative, but it also disqualifies some interactions completely, thus acting as a form of 'filter' which will produce 'cleaner' results; we thus henceforth adopt the Holm scheme. 

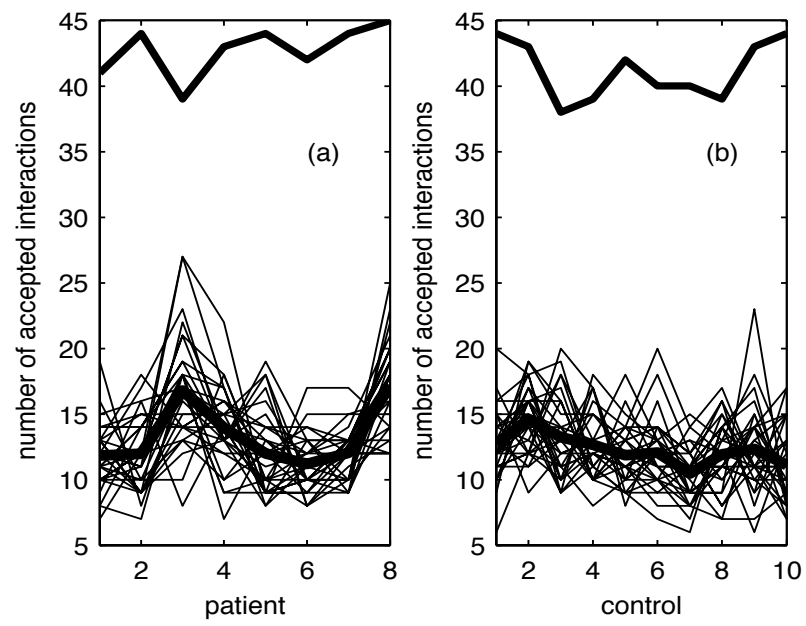

Figure 9: A comparison of interaction results for multiple epochs with the result of averaging spectral matrices over epochs. (a) patients, and (b) controls. The Holm procedure was used in hypothesis testing. See text for details.

\section{The question of averaging}

Since we have multiple patients and controls, and multiple epochs for each, should we first create average spectral matrices by averaging matrices over individuals and/or epochs, and then carry out hypothesis testing? This could be justified if we could make the assumption that the matrices were independent and identically distributed so that the overall average was an estimate of a well-defined quantity. Also the number of degrees of freedom for the tests, $n$, would simply be $K$ multiplied by the number of individuals and/or epochs.

Consider averaging over epochs. While the relatively short epochs can individually be treated as stationary segments, the long unsegmented series is not stationary, so that the stationary segments are not samples from the same stationary process and therefore should not be treated as such. Even if they were treated as such, there is still a problem. Some epochs are contiguous, and some are not since an epoch (across all 10 channels) is removed if there is a departure in excess of $\pm 100 \mu \mathrm{V}$ in one of the channels, (see Section 3.2). Hence the level of correlation between epochs for an individual, albeit likely to be quite small, is variable and unquantifiable and the resulting degrees of freedom are indeterminate. In Fig. 9 the ensemble of thin lines show the number of accepted interactions for the different epochs for (a) patients and (b) controls, and the thick line amongst them is the average of the ensemble. We see that the number of interactions seems less variable with individual controls than it does for individual patients. The thick line at the top of each plot is the result obtained if spectral averaging is first carried out over the epochs, with the number of degrees of freedom taken to be $30 K$, the product of the number of epochs and the number of tapers, i.e., independence of epochs is assumed.
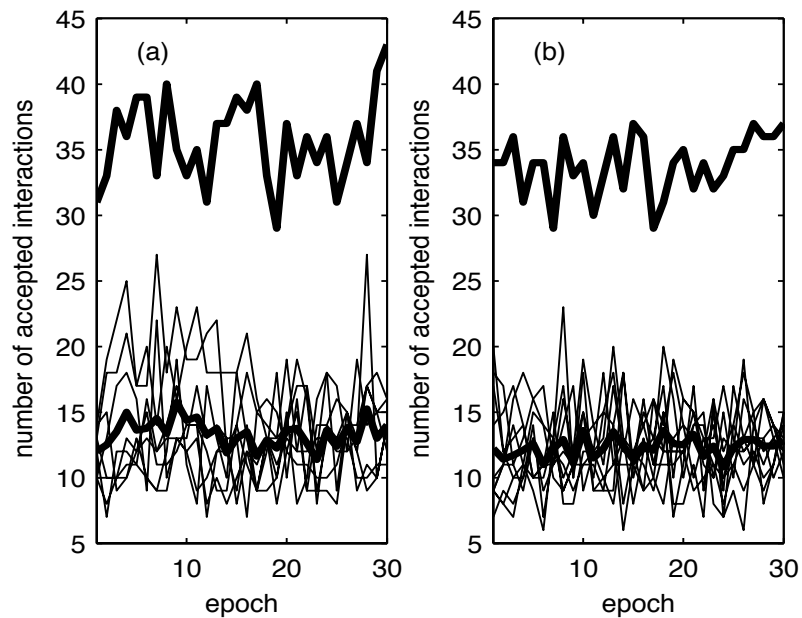

Figure 10: A comparison of interaction results for multiple individuals with the result of averaging spectral matrices over individuals. (a) patients, and (b) controls. The Holm procedure was used in hypothesis testing. See text for details.

The results with and without averaging are clearly inconsistent.

Averaging over individuals for the same epoch should not be affected by lack of independence, but there is no $a$ priori reason to expect that interaction effects should manifest themselves in terms of identically-distributed spectral matrices at any frequency $f$. For example the largest partial coherence - possibly leading to rejection of the null hypothesis - could occur at nearby but different frequencies for two individuals. Averaging the spectral matrices will mix-up these effects. Also, the phases on the signals recorded for different individuals will differ, so that averaging will mix up these effects too. In Fig. 10 the ensemble of thin lines show the number of accepted interactions for the individuals for (a) patients and (b) controls, and the thick line amongst them is again the average of the ensemble. The number of interactions seems slightly more variable with controls than patients, but the average line is reasonably constant in both cases. The thick line at the top of each plot is the result obtained if spectral averaging is first carried out over the individuals, with the number of degrees of freedom taken to be the product of the number of tapers and the number of patients or controls, i.e., independence of individuals is assumed. The results with and without averaging are again inconsistent, but slightly less so than for averaging over epochs.

We hence chose to keep our interaction analysis results on a per individual per epoch basis, and consider how these could be sensibly combined.

\section{Determining connectivity}

\subsection{Basic steps}

So far we have decided on the number of tapers to use $(K=20)$ via the technique of 'window closing,' used spec- 

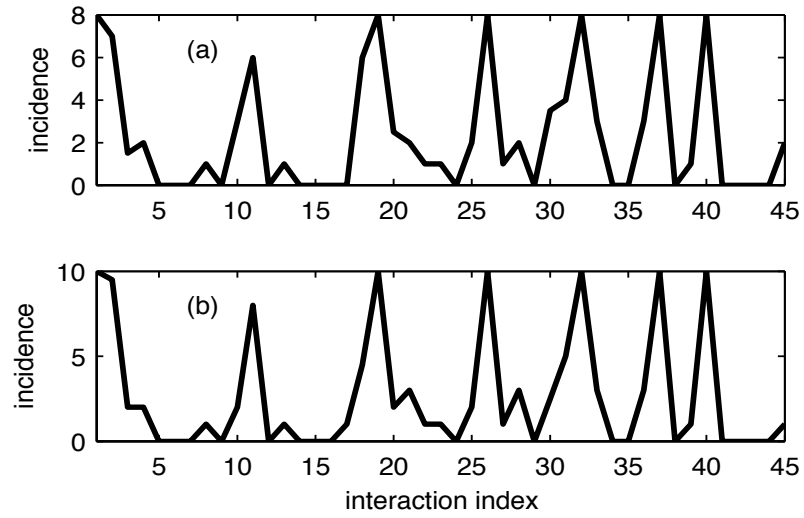

Figure 11: The median number of individuals over epochs for which one or more partial coherencies are non-zero, as a function of interaction index for (a) patients and (b) controls, both for Holm scheme.

tral line removal and reshaping to negate the 'power line pick-up,' mean-corrected and rescaled the epochs to unity standard deviation, and considered multiple testing procedures for which FWER $\leq \alpha$.

\subsection{Relative strength}

The theory of time series graphical modelling (Dahlhaus, 2000) states that an interaction exists if the partial coherence is non-zero at one or more frequencies. From a practical perspective we might like to distinguish between partial coherencies which are non-zero over multiple frequencies and those which exhibit a spike (perhaps spurious) at one odd frequency. This is the role of $R R H$ in (21). We might usefully combine this with the number of individuals, for patients or controls, exhibiting certain interactions. Fig. 11 shows the median number of individuals over epochs for which $R R H>0$, i.e., one or more partial coherencies are non-zero. Some interactions are found to be present in a very large proportion of the individuals - in several cases all of them - whereas some interactions have a very low or zero incidence.

We could have the situation that a particular interaction has a large $R R H$ for only a very small proportion of individuals (low incidence). Vice versa, if an interaction is present in most patients or controls, but with negligible strength, one might be suspicious of undetected interference at a particular frequency. We could consider both measures simultaneously via the product

$$
\overline{R R H} \cdot \overline{P I},
$$

where $\overline{P I}$ denotes the median over epochs of the proportion of individuals (patients or controls) exhibiting the interaction. We call the product (22) the relative strength of the interaction.

The values of relative strength for the interactions are given in Table 3, with the upper triangle giving the results for patients, and the lower triangle those for controls. We

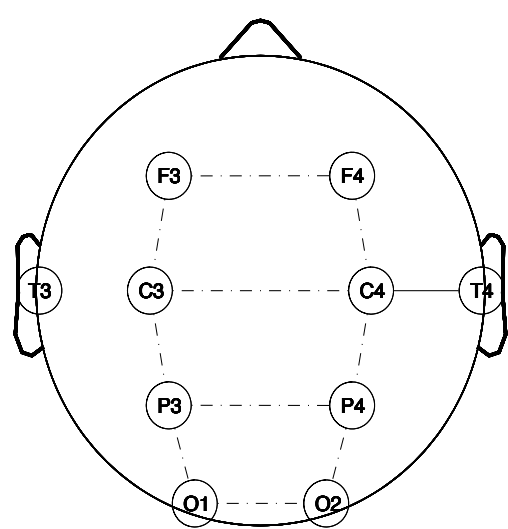

Figure 12: Graphical display of interactions based on the relative strengths shown in Table 3. (See the text for display details.) Dependent Holm scheme used for hypothesis testing.

notice that the results are for the most part very symmetric in terms of relative strengths for patients and controls which would seem to reflect on the stability of the methodology. The non-zero connections identified in Table 3 are plotted in Fig. 12 where the dash-dot lines are connections common to both patients and controls. The solid line marks the $\mathrm{C} 4$-T4 connection found only in patients; however, from Table 3 we see that its relative strength is only 0.0002. We also see from Table 3 that the F3-F4 connection is strongest, as would have been expected from partial coherencies such as in Fig. 7(a) which as we mentioned earlier were widely observed. The C3-C4 and P3-P4 connections appear quite weak, and the F3-C3 and F4-C4 connections only a little stronger.

\section{Discussion}

It has been demonstrated in this paper that the formulation of a graphical brain connectivity model via partial coherence analysis contains several facets that must be carefully considered and have the potential to considerably influence the outcome. These include the degree of spectral smoothing, line and interference removal, matrix inversion stabilization and side-lobe leakage suppression, the combination of results from different epochs and people, and multiple hypothesis testing. We have examined each of these steps in turn and provided a possible path which produces relatively 'clean' connectivity plots.

Connectivity in medicated schizophrenia patients and controls appears very similar, based on our EEG data and the Holm hypothesis testing procedure. In future work we intend to apply our methodologies to other patient groups, and to particular frequency bands to gain further insight on possible differences in connectivity. 


\begin{tabular}{|c||c|c|c|c|c|c|c|c|c|c|}
\hline & F3 & F4 & C3 & C4 & P3 & P4 & O1 & O2 & T3 & T4 \\
\hline \hline F3 & & 0.40 & 0.064 & 0 & 0 & 0 & 0 & 0 & 0 & 0 \\
\hline F4 & 0.34 & & 0 & 0.020 & 0 & 0 & 0 & 0 & 0 & 0 \\
\hline C3 & 0.069 & 0 & & 0.014 & 0.17 & 0 & 0 & 0 & 0 & 0 \\
\hline C4 & 0 & 0.023 & 0.0002 & & 0 & 0.13 & 0 & 0 & 0 & 0.0002 \\
\hline P3 & 0 & 0 & 0.17 & 0 & & 0.0007 & 0.14 & 0 & 0 & 0 \\
\hline P4 & 0 & 0 & 0 & 0.16 & 0.0015 & & 0 & 0.11 & 0 & 0 \\
\hline O1 & 0 & 0 & 0 & 0 & 0.10 & 0 & & 0.13 & 0 & 0 \\
\hline O2 & 0 & 0 & 0 & 0 & 0 & 0.10 & 0.10 & & 0 & 0 \\
\hline T3 & 0 & 0 & 0 & 0 & 0 & 0 & 0 & 0 & & 0 \\
\hline T4 & 0 & 0 & 0 & 0 & 0 & 0 & 0 & 0 & 0 & \\
\hline
\end{tabular}

Table 3: Relative strengths for the interactions. Results for patients in upper triangle, and for controls in the lower triangle. (Holm hypothesis testing used.)

\section{Acknowledgements}

This work was supported via a grant from the Engineering and Physical Science Research Council (UK). Helpful suggestions by three reviewers were much appreciated and improved the exposition considerably.

\section{References}

Bendat, J. S. Statistical errors in measurement of coherence functions and input/output quantities. Journal of Sound and Vibration., 1978; 59: 405-21.

Brillinger, D. B. Second-order moments and mutual information in the analysis of time series. In: Chaubey YP, editor. Recent Advances in Statistical Methods. London: Imperial College Press, 2002: 64-76.

Carlson, B. D. Covariance matrix estimation errors and diagonal loading in adaptive arrays. IEEE Trans. on Aerospace and Electronic Systems, 1988; 24: 397-401.

Celka, P. [Ed.] Special issue on Neuronal Coordination in the Brain: A Signal Processing Perspective. Signal Processing, 2005: 85, no. 11.

Cohen, M. I., Yu, Q. \& Huang, W.-X. Preferential correlations of medullary neuron's activity to different sympathetic outflows as revealed by partial coherence analysis. J. Neurophysiol., 1995; 74: 474-8.

Dahlhaus, R. Graphical interaction models for multivariate time series. Metrika, 2000; 51: 157-72.

Dahlhaus, R., Eichler, M. and Sandkühler, J. Identification of synaptic connections in neural ensembles by graphical models. J. Neurosci. Meth., 1997; 77: 93-107.

Delorme, A. and Makeig, S. EEGLAB: an open source toolbox for analysis of single-trial EEG dynamics including independent component analysis. J. Neurosci. Meth., 2004; 134: 9-21.

de Boer P. Ridge regression revisited. Statistica Neerlandica, 2005; 59: 498-505.

Eichler, M. Graphical Models in Time Series Analysis. PhD dissertation, Ruprecht-Karls-Universität Heidelberg, 1999.

Granger, C. W. J. and Hatanaka, M. Spectral Analysis of Economic Time Series. Princeton, NJ: Princeton University Press, 1964.

Halliday, D. M. Spike-train analysis for neural systems. In Reeke GN, Poznanski RR, Lindsay KA, Rosenberg JR, Sporns O, editors. Modeling in the Neurosciences, second ed. Taylor \& Francis, 2005, 555-79.

Hämäläinen, M., Nolte, G., Worsley, K., de Munck, J. C. [Eds.] Special Section on Signal Processing Aspects of Brain Imaging. IEEE Trans. Signal Proc., 2005; 53, no. 9.
Holm, S. A simple sequentially rejective multiple test procedure. Scandinavian Journal of Statistics, 1979; 6: 65-70.

Larsen, P. D., Lewis, C. D., Gebber. G. L. \& Zhong, S. Partial spectral analysis of cardiac-related sympathetic nerve discharge. J. Neurophysiol., 2000; 84: 1168-79.

Lehmann, E. L. and Romano, J. P. Testing Statistical Hypotheses, 3rd Ed. New York: Springer, 2005.

Mima, T., Matsuoka, T. \& Hallett, M. Functional coupling of right and left cortical motor areas demonstrated with partial coherence analysis. Neurosci. Lett., 2000; 287: 93-6.

Percival, D. B. and Walden, A. T. Spectral Analysis for Physical Applications. Cambridge UK: Cambridge University Press, 1993.

Rosenberg, J. R., Amjad, A. M., Breeze, P., Brillinger, D. R. and Halliday, D. M. The Fourier approach to the identification of functional coupling between neuronal spike trains. Progress in Biophysics and Molecular Biology, 1989; 53: 1-31.

Rosenberg, J. R., Halliday, D. M., Breeze, P. and Conway, B. A. Identification of patterns of neuronal connectivity - partial spectra, partial coherence, and neuronal interactions. J. Neurosci. Meth., 1998; 83: 57-72.

Salvador, R., Suckling, J., Schwarzbauer, C. and Bullmore, E. Undirected graphs of frequency-dependent functional connectivity in whole brain networks. Phil. Trans. Roy. Soc. Lond., Ser. B, 2005; 360(1457): 937-46.

Strang G. Linear Algebra and its Applications., third ed. Harcourt Brace Jovanovich: Orlando FL, 1988.

Valdés-Sosa, P. A., Kötter, R. and Friston, K. J. [Eds.] Theme Issue on Multimodal Neuroimaging of Brain Connectivity. Phil. Trans. Roy. Soc. Lond., Ser. B, 2005; 360 (1457).

Walden, A. T. A unified view of multitaper multivariate spectral estimation. Biometrika, 2000; 87: 767-87.

Walden, A. T., McCoy, E. J. \& Percival, D. B. The effective bandwidth of a multitaper spectral estimator. Biometrika, 1995; 82: 201-14. 